\title{
Spontaneous redox route for gold-aryl film development of latent fingerprints on nickel coins
}

\author{
Saeed Almheiri, ${ }^{a, b}$ Ahmad A. L. Ahmad, ${ }^{c}$ Benjamin Le Droumaguet, ${ }^{b}$ Rémy Pires, ${ }^{b}$ \\ Mohamed M. Chehimi, ${ }^{\text {, }}$ Ahmed A. Mohamed ${ }^{\mathrm{a}, *}$
}
a. Center for Advanced Materials Research, Research Institute of Sciences and Engineering, University of Sharjah, Sharjah 27272, UAE
b. Univ Paris Est Créteil, CNRS, UMR 7182, UPEC, F-94320 Thiais, France
c. Department of Chemistry, University of Maine, Orono, Maine 04469, United States

Corresponding Authors

*E-mail: chehimi@icmpe.cnrs.fr. (M.M.C.)

*E-mail: ah.mohamed@sharjah.ac.ae. (A.A.M.)

ORCID

Ahmad A. L. Ahmad: 0000-0003-3271-326X

Benjamin Le Droumaguet: 0000-0002-5757-3606

Mohamed M. Chehimi: 0000-0002-6098-983X

Ahmed A. Mohamed: 0000-0001-7369-3117 


\begin{abstract}
Surface modifiers through redox routes emerged as the most preferred in forensic science. Aryldiazonium salts stabilized with tetrachloroaurate(III) are excellent redox modifiers in the development of latent fingerprints on nickel surfaces. Nickel coins were fingerprinted and developed with a stable diazonium salt $\left[\mathrm{O}_{2} \mathrm{~N}-4-\mathrm{C}_{6} \mathrm{H}_{4} \mathrm{~N} \equiv \mathrm{N}\right] \mathrm{AuCl}_{4}$. The developed surfaces were studied using XPS which showed the presence of the characteristic gold and organic modifier peaks. In addition, imaging analysis using SEM showed clusters of gold. EDS quantitative analysis estimated a good amount of gold presence on the fingerprinted compared to the nonfingerprinted area. Our results are important in the analysis of nickel coins using a spontaneous route by simply depositing diazonium salt solution drops on the nickel surface without any additional reducing agent. This work brings strong supporting evidence for the efficient combination of diazonium surface chemistry and high-performance surface analytical tools (XPS, SEM-EDS) in the fast, spontaneous developing of fingerprints and their morphological and chemical composition assessments.
\end{abstract}

Keywords. Forensics, Diazonium Gold Salt, Nickel Coins, XPS, Latent Fingerprints, Gold-Aryl Film. 


\section{Introduction}

Redox approaches in forensic science investigation emerged as the preferred choice for surface chemists. ${ }^{1-3}$ Reducible gold(III) precursors stabilized with an organic moiety such as diazonium have been stabilized and developed in forensic science to be handled at ambient conditions. ${ }^{4}$ Their enhanced solubility made them the first choice in developing metals and nonmetals. They are prone to spontaneous redox reactions without the need for electrochemical or chemical stimuli. They also form robust metal-carbon bonding which persists under environmental conditions. ${ }^{5-10}$ Recently, we investigated aryldiazonium tetrachloroaurate(III) salts as developers for latent fingerprints collected on several metals such as aluminum, lead and copper. ${ }^{11}$ Compiled data suggested that the salts are excellent modifiers on copper surfaces but no other metals due to their high reduction ability which resulted in poor quality development over all fingerprint. The cleavage of molecular nitrogen as bubbles and the immediate development can be seen. XPS study on copper surfaces resulted in the formation of gold(0) accompanied by copper oxide formation. ${ }^{11}$

Nickel-diazonium interface has been of central interest to investigate which can spontaneous reduce the diazonium salts by just simple dipping, induction heating or electrochemical reduction with low oxidation potential $\left(-0.257 \mathrm{~V}\right.$ vs. SHE) ${ }^{6,13-15}$ The resulting attached organic layer shows high stability and resistance of sustained ultrasonic rinsing in different solvents. ${ }^{7}$

Nickel-plated brass is one of the major types of casings encountered in the market cartridge

casing. ${ }^{16,17}$ It was used primarily as a method of preventing the verdigris and corrosion to brass ammunition stored in leather cartridge belts and bags. In coins, nickel alloys were used in many countries and regions worldwide such the United States, ${ }^{18}$ UK coins, ${ }^{19}$ Euro, and Sweden. ${ }^{20}$ 
Nickel has been investigated in forensic science in counterfeit watches and coins, ${ }^{21-23}$ human teeth involved crimes,${ }^{24}$ illicit cocaine, ${ }^{25}$ prints on cartridge casings and shotgun shells ${ }^{26}$ and

cosmetic evidence. ${ }^{27}$ Several techniques were used in the analysis such as XPS, XRF, XRD, ICPMS and simple chemical modification. ${ }^{22,25,28-34}$

Herein, we bridge the gap between previous works on diazonium modification of copper and our approach applying diazonium gold to surfaces and their analyses by XPS to tackle the latent fingerprint development on nickel coins. We describe our results of spontaneous redox development of nickel surfaces using nitrobenzenediazonium tetrachloroaurate(III) (NBD $\mathrm{AuCl}_{4}$ ) and studied using XPS, SEM and EDX. Nickel surfaces are more reducing than copper for example and expected to reduce diazonium salts faster and more efficiently; this is what motivated our choice of one French Franc nickel coin to conduct the study within the framework of forensic surface chemistry and surface analysis.

\section{Materials and methods}

\subsection{Instruments}

A K Alpha XPS apparatus (Thermo Scientific) was used to record the spectra. The machine is fitted with a monochromatic $\mathrm{Al} \mathrm{K \alpha} \mathrm{X}$-ray source (spot size: $100 \mu \mathrm{m}$ ). The pass energy was set to 80 and $200 \mathrm{eV}$ for the narrow regions and survey spectra, respectively. Electron and argon flood guns were used to compensate for the static charge buildup. It is to note that charge neutralization was not necessary for cleaned nickel coin; but was applied in order to have similar analysis conditions for metal surface and the same after reaction with diazonium salt which reacts with- and acrylates the surface in addition to the deposition of gold. Metallic state nickel $\mathrm{Ni} 2 \mathrm{p}_{3 / 2}$ peak was found at $852.9 \mathrm{eV}$ which is the value reported in NIST database for conductive nickel surface (https://srdata.nist.gov/xps/XPSDetailPage.aspx?AllDataNo=26452 ); in this case 
no further calibration was needed. For coated surfaces, gold Au4f7/2 peak was found at $84 \pm 0.1$

eV. The composition was determined using the manufacturer sensitivity factors. SEM investigations were performed using a Carl Zeiss MERLIN field emission-scanning electron microscope (FE-SEM). In order to reveal alternating section in composition of the surface, backscattered electrons (BSE) were collected and imaged. Energy dispersive X-ray analysis (EDS) were performed using a X-Max silicon drift detector from Oxford Instruments coupled with AZtec operating system.

\section{2. $\mathrm{NO}_{2}$-aryldiazonium-AuCl 4 on glass slides}

Surface preparation. Glass slides were cut into five pieces and prepared by cleaning with $\mathrm{HCl}$ solution to remove metal-based contamination, as well as $\mathrm{NaOH}$ to remove organic contaminants, then absolute ethanol.

Developing latent fingerprint on non-metal surface. The following samples were prepared for XPS analysis: Glass (clean); Glass + Fingerprint. The aryldiazonium $\mathrm{AuCl}_{4}$ solution $(0.001 \mathrm{M})$ was dropped using a dropper on each of the prepared surfaces: Glass ( 2 h); Glass + Fingerprint $(1 \mathrm{~h})$; Glass + Fingerprint $(2 \mathrm{~h})$. The surfaces were then washed gently with deionized water until the excess salt was completely washed away and dried.

\section{3. $\mathrm{NO}_{2}$-aryldiazonium-AuCl 4 on nickel coins}

Surface preparation. Nickel coins (French Franc) were prepared by cleaning with distilled white vinegar and table salt to remove the oxides, sonification to remove any dirt, then absolute ethanol.

Developing latent fingerprint on metal surface. The following samples were prepared for XPS analysis: One French Franc nickel coin (clean). The aryldiazonium $\mathrm{AuCl}_{4}$ solution 
$(0.001 \mathrm{M})$ was dropped using a dropper on each of the prepared surfaces: Nickel coin $(1 \mathrm{~h})$; Nickel coin + Fingerprint $(1 \mathrm{~h})$. The surfaces were then washed gently with deionized water until the excess salt was completely washed away and dried. The samples formed a film just enough to cover the suspected area.

\section{Results and Discussion}

The demonstration of latent fingerprints on glass slides were tested as representative materials of windows and glass bottles. However, no conclusive results were obtained after $2 \mathrm{~h}$ of interactions between diazonium salt solution drops and glass samples with fingerprints (no visible fingerprints were noted). This is probably due to the lack of non-reducing nature of glass. It is to

note that in this work, we have used a minimum set of compounds; we simply deposited diazonium salt solution drops on the surface without any additional reducing agent. This might be the reason latent fingerprint could possibly be demonstrated on glass, via a spontaneous redox process. Indeed, it was reasonable to expect possible reaction between diazonium group and silanol groups resulting in $\mathrm{Si}-\mathrm{O}-\mathrm{N}=\mathrm{N}$-aryl interface.

Samples consisted of clean nickel coin, nickel coin treated with diazonium, nickel coin coated with fingerprint and post-treated with diazonium were analyzed using XPS (Fig. 1a-d). A fingerprint sample of the right thumb was collected. We differentiate the fingerprint side and the non-fingerprint side of the nickel sample by looking at the survey scan and observing the shape of the background between $600-900 \mathrm{eV}$ on the binding energy scale. In this region, the inelastic background is ascending from the lower to the higher binding energy side, therefore testifying for a more screened substrate due to the co-existence of both the fingerprint and the diazonium reaction products (Fig. 1a). This is the same situation if one inspects the narrow Ni2p region 
(Fig. 1b); it shows a higher inelastic background at high binding energy side (low kinetic energy side).

Ni2p ( 852.9 and $855.3 \mathrm{eV})$ from the nickel coin (Fig. 1b), indicates the co-existence of both nickel in the metallic and the oxidized states, respectively. Indeed, the main $\mathrm{Ni} 2 \mathrm{p}_{3 / 2}$ peak from the oxide is broad and has a satellite characteristic of Ni(II). Au4f (Fig. 1c) and N1s (Fig. 1d) correspond to diazonium and/or the fingerprints. N1s region (from Ni coin with fingerprint and diazonium) has two main peaks at about 399.8 and $405.7 \mathrm{eV}$ assigned to $-\mathrm{N}=\mathrm{N}-$ from the aryl groups within the aryl layer and the fingerprints, and the $\mathrm{NO}_{2}$ group from the diazonium, respectively (Fig. 1d). In samples containing fingerprints, Ni2p has the lowest atomic percent because, compared to cleaned, bare coins, the nickel is attenuated by both the fingerprint and the aryl/gold deposits. Therefore, we no longer observe the components due to N1s from the diazonium group $\mathrm{N}_{2}{ }^{+} ;{ }^{12}$ this accounts for the spontaneous reduction of the diazonium cation on nickel, a result that is in line with the spontaneous arylation of nickel reported for $\mathrm{NBD} \mathrm{BF}_{4}{ }^{7}$ These XPS results, demonstrating attenuation of N1s from diazonium compound, parallel the direct observation of bubbles (Fig. 1e) when a drop of diazonium solution is gently deposited on nickel with or without fingerprint. Au4f $\mathrm{f}_{7 / 2}$ (Fig. 1c), centered at $\sim 84.1 \mathrm{eV}$, accounts for metallic state gold (NIST XPS database, https://srdata.nist.gov/xps/) and thus a reductive process affecting $\mathrm{AuCl}_{4}{ }^{-}$anions. 

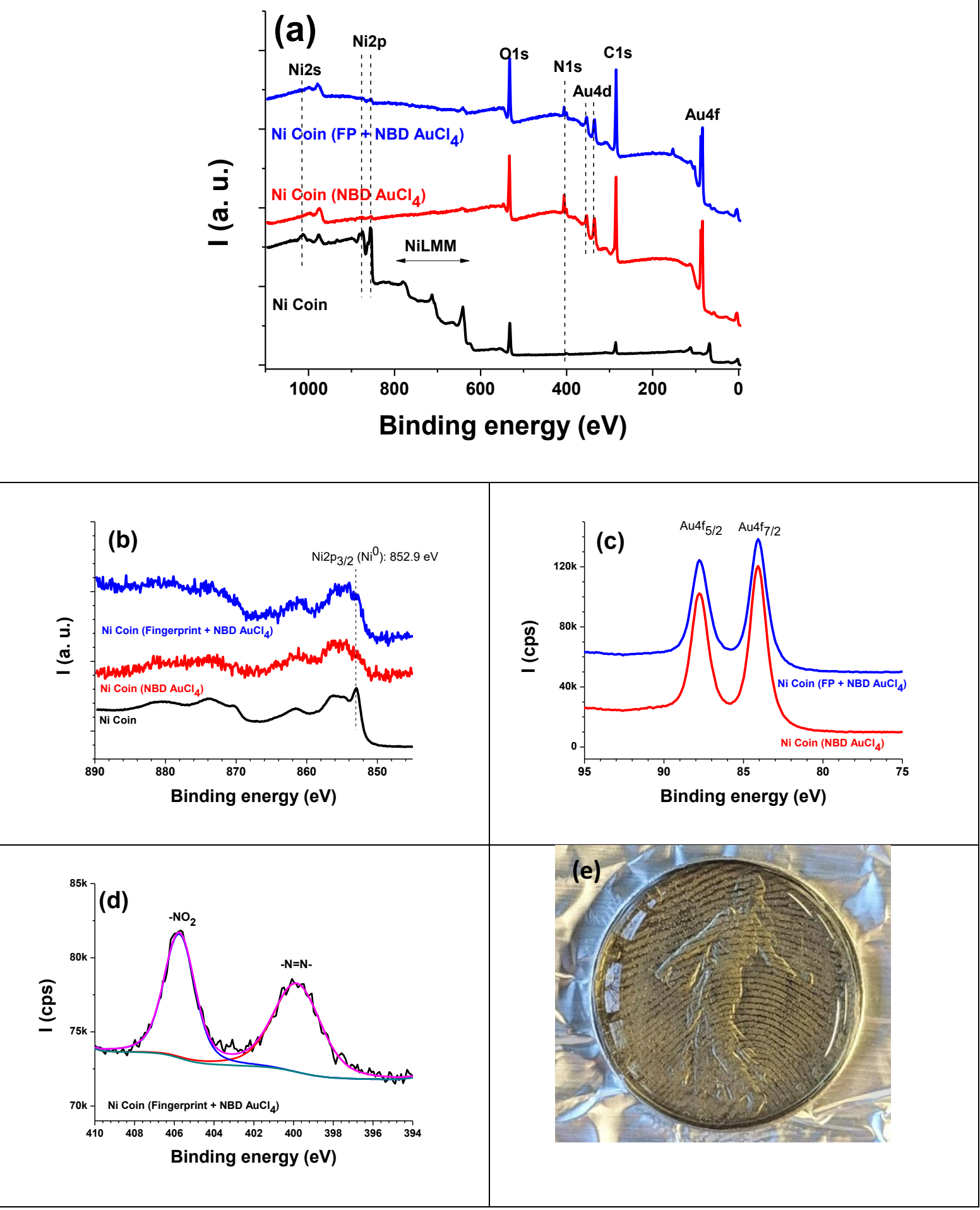

Fig. 1. (a) XPS survey scan of fingerprint and $\mathrm{NO}_{2}$-aryldiazonium- $\mathrm{AuCl}_{4}$ treated nickel sheet, (b) high-resolution Ni2p, (c) and Au4d spectra, (d) peak-fitted N1s from the coin with diazonium visualized fingerprint, and (e) difital photograph of latent fingerprint demonstration using $\mathrm{NO}_{2}-$ aryldiazonium- $\mathrm{AuCl}_{4}$ solution on nickel coin. N1s peak fitting parameters for $\mathrm{N} 1 \mathrm{~s}(\mathrm{azo})$ and $\mathrm{N} 1 \mathrm{~s}$ $\left(\mathrm{NO}_{2}\right)$ : peak position $=399.8 / 405.7 \mathrm{eV} ; \mathrm{FWHM}=2.8 / 1.85 \mathrm{eV}$. 
The surface compositions of materials are reported in Table 1. The table considers the unique elemental markers of the diazonium compound and the nickel substrate. Diazonium reacts to yield metallic gold and aryl groups represented by the unique $\mathrm{NO}_{2}$ type of nitrogen. $\mathrm{Ni} 2 \mathrm{p}$ accounts for both the metallic and oxidized states of nickel. We report $\mathrm{NO}_{2} / \mathrm{Ni}$ and $\mathrm{Au} / \mathrm{Ni}$ atomic ratios on the one hand, and the absolute $\mathrm{Ni}$ at.\%, on the other hand. It is clear that the total nickel content drastically decreases at the surface after placing the fingerprint and further reacting the substrate with the diazonium salt. The fingerprint area shows lower $\mathrm{NO}_{2}$ and gold relative to the total nickel. This point will best be discussed in the light of the SEM-EDS characterization.

Table 1. Apparent elemental composition of nickel coin on different treatments (in at.\%).

\begin{tabular}{|c|c|c|c|}
\hline Material & $\mathrm{NO}_{2} / \mathrm{Ni}$ & $\mathrm{Au} / \mathrm{Ni}$ & $\mathbf{N i}$ \\
\hline Ni coin (clean) & 0 & 0 & 20.6 \\
\hline $\mathrm{Ni}$ coin $\left(\mathrm{NO}_{2}\right.$-Aryldiazonium- $\left.\mathrm{AuCl}_{4}\right)$ & 9.56 & 4.02 & 2.14 \\
\hline $\mathrm{Ni}$ coin (Fingerprint $+\mathrm{NO}_{2}$-Aryldiazonium- $\mathrm{AuCl}_{4}$ ) & 4.53 & 3.33 & 2.30 \\
\hline
\end{tabular}

Further SEM images confirm the elemental composition of the surfaces (Fig. 2 and 3). The SEM images of non-fingerprint area (Fig. 2-a) show a thin gold/aryl sheet layer on top of nickel metal, with a minor case of gold nanoclusters. Fingerprint area (Fig. 3) shows a high amount of gold nanoclustering (Fig. 3b,e) on top of nickel metal, with a minor case of gold/aryl sheet. The EDS spectra displayed account for the SEM images of both types of surfaces. 

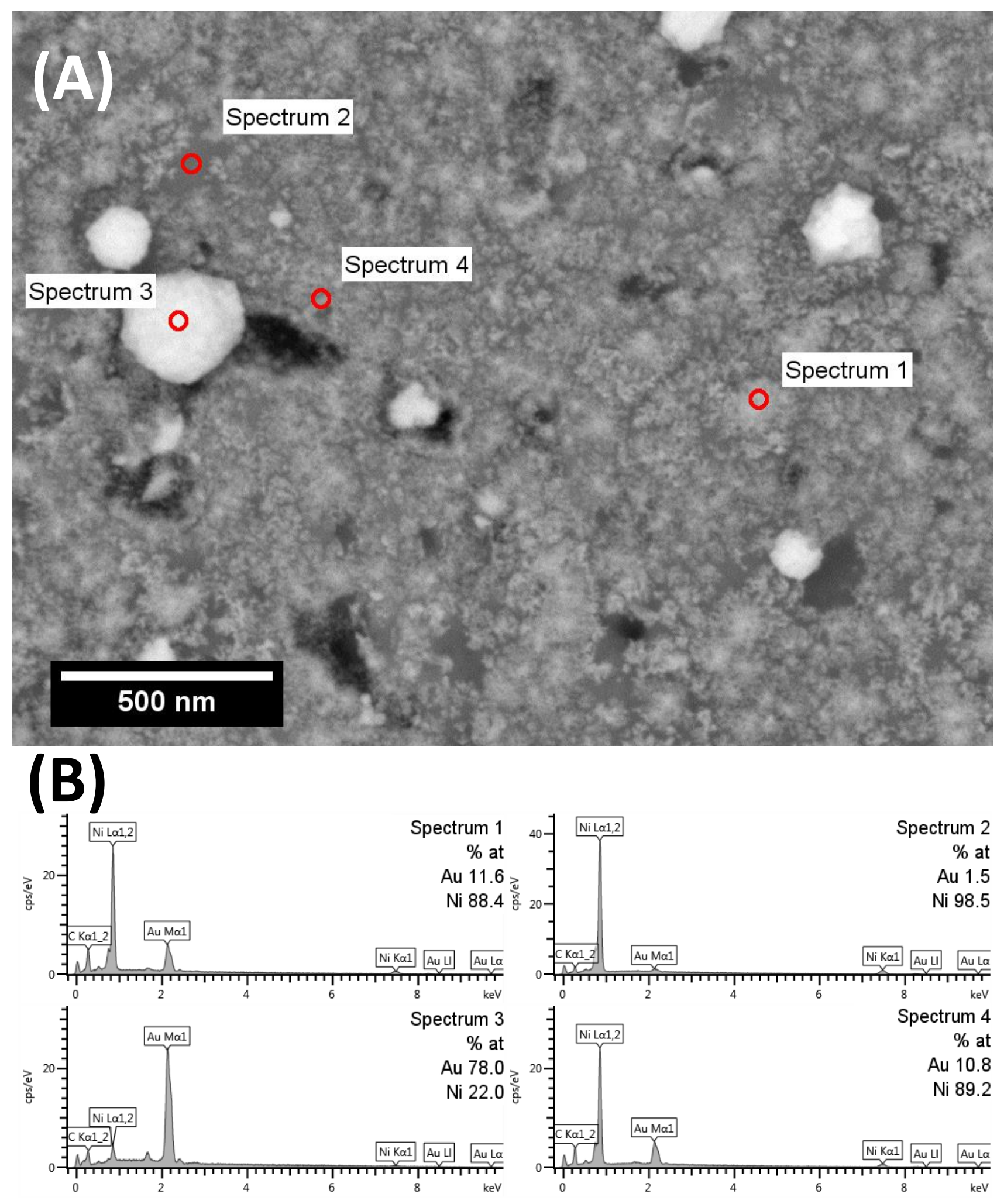

Fig. 2. SEM image (A) and elemental spectra (B) of the non-fingerprint area of treated nickel coin with $\mathrm{NO}_{2}$-aryldiazonium-AuCl 4 . Spectra 1-4 in (B) were taken from red circles shown in (A). 

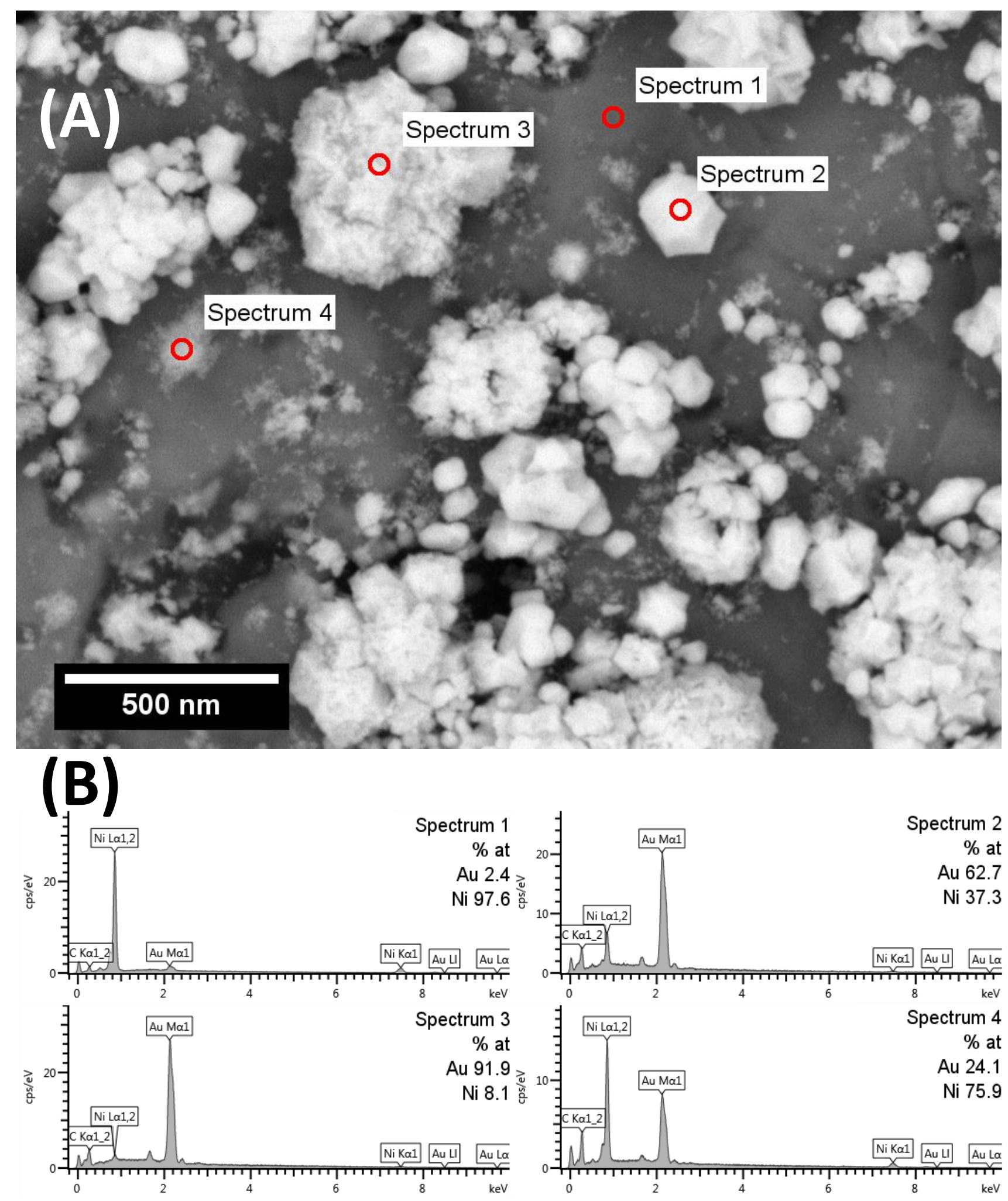

Fig. 3. SEM image (A) and elemental spectra (B) of the fingerprint area of treated nickel coin with $\mathrm{NO}_{2}$-aryldiazonium- $\mathrm{AuCl}_{4}$. Spectra 1-4 in (B) were taken from red circles shown in (A). 
These results were compatible with our recent work using the gold aryldiazonium salt on the copper coin surface. ${ }^{6}$ The fingerprint treatment on the copper coin with the diazonium salt solution showed a clear sign of $\mathrm{Cu}(\mathrm{II})$, measured by the XPS technique, which gives the black color on the coin. This combination of fingerprints and the aryldiazonium salt allow the visualization of the latent fingerprints. Also, high quality images were observed on copper and lead surfaces using the same treatment process. ${ }^{11}$ The latent fingerprint produced aided to visualize the details for the three levels of primary, secondary and tertiary fingermarks studies.

Combining all results discussed above, it is possible to schematically illustrate the situation of this latent fingerprint development (Fig. 4). Direct application of the diazonium salt solution drop on the bare nickel yields a thin coating, whereas the application of the same solution drops on the fingerprints induces aryl coating topped with gold clustering.

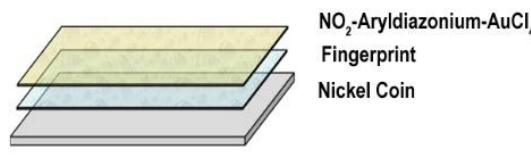

Fingerprint (light) region
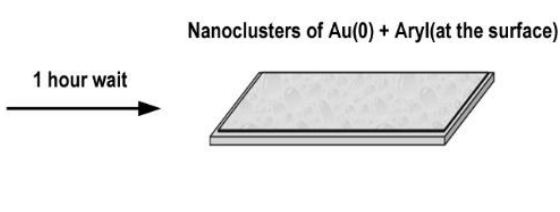


\section{Conclusions}

$\mathrm{NO}_{2}$-aryldiazonium- $\mathrm{AuCl}_{4}$ salt solution was prepared and applied on nickel and glass surfaces. The samples were first treated with the salt solution for a measured time without any application of fingerprints and subsequently analyzed with XPS. Treated nickel samples produced $\mathrm{N}_{2}$ bubbles on the non-fingerprint side and light lines on the fingerprint side. Treated glass did not react due to its non-reducible nature. For comparison, in $\mathrm{Cu}$ sample analysis, there is an indication of copper leaching through the fingerprint and gold/aryl coating, whereas in nickel sample analysis, Au tends to create nanocluster on fingerprints deposited area. Particularly on nickel surface, Au and salt solution creates a film which covers the Ni surface in the absence of fingerprints. This observation indicates the potential application of $\mathrm{Au}(\mathrm{III})$ salt solution for better detection of latent fingerprints.

This experiment highlights the unique combination of high-performance surface analytical tools (XPS, SEM and EDS) and diazonium chemistry to study the development of latent fingerprints without any external action (for example without any applied potential as done elsewhere). Aryldiazonium gold(III) salts offer fast application of fingerprints detection method and can be further applied to other systems of metal surfaces that have a reducing power.

Beyond this work, if aryldiazonium salts proved to be important compounds for surface modification in general, aryldiazonium gold(III) salts appear as unique compounds of paramount importance in "forensic surface chemistry."

\section{Conflict of interest}

None 


\section{Acknowledgements}

A.A.M. acknowledges the University of Sharjah support of SEED grant VC-GRC-SR-832015, competitive grants 160-2142-029-P and 150-2142-017-P, Organometallic Research Group grant RISE-046-2016, and Functionalized Nanomaterials Synthesis Lab grant 151-0039. S.A.A. thanks the General Department of Forensic Science and Criminology (Dubai Police HQ, Dubai) for financial support to visit ICMPE and perform surface modification and XPS analyses. 


\section{References}

1. Bond JW. The thermodynamics of latent fingerprint corrosion of metal elements and alloys. J. Forensic Sci. 2008;53:1344-1352.

2. Bécue A. Emerging fields in fingermark (meta)detection - a critical review. Anal. Methods. 2016;8:7983-8003.

3. Raicopol M, Necula L, Ionita M, Pilan L. Electrochemical reduction of aryl diazonium salts: a versatile way for carbon nanotubes functionalisation. Surf. Interface Anal. 2012;44:1081-1085.

4. Mohamed AA. Gold is going forensic. Gold Bull. 2011;44:71-77.

5. Chehimi MM. Aryl Diazonium Salts: New Coupling Agents in Polymer and Surface Science, Wiley-VCH: Verlag GmbH \& Co. KGaA, 2012.

6. Mesnage A, Lefèvre X, Jégou P, Deniau G, Palacin S. Spontaneous grafting of diazonium salts: chemical mechanism on metallic surfaces. Langmuir. 2012;28:11767-11778.

7. Adenier A, Barre N, Cabet-Deliry E, Chaussé A, Griveau S, Mercier F, Pinson J, Vautrin-Ul C. Study of the spontaneous formation of organic layers on carbon and metal surfaces from diazonium salts. Surf. Sci. 2006;600:4801-4812.

8. Orefuwa A, Ravanbakhsh M, Neal SN, King JB, Mohamed AA. Robust organometallic gold nanoparticles. Organometallics. 2014;33:439-442.

9. Tuyet VBT, Cannizzo C, Legros C, Andrieux M, Chaussé A. Modification of fluorinedoped tin oxide surface: Optimization of the electrochemical grafting of diazonium salt, Surfaces and Interfaces. 2019;15:110-116.

10. Jasmin JP, Miserque F, Dumas E, Vickridge I, Ganem JJ, Cannizzo C, Chaussé A. XPS and NRA investigations during the fabrication of gold nanostructured functionalized screen-printed sensors for the detection of metallic pollutants, Appl. Surf. Sci. 2017;397:159-166.

11. Ahmad AAL, Alawadhi AH, Park J, Abdou HE, Mohamed AA. Evaluation of diazonium gold(III) salts in forensic chemistry: Latent fingerprint development on metal surfaces. Forensic Chem. 2019;13:100144.

12. Almheiri S, Ahmad AAL, Le Droumaguet B, Pires R, Mohamed AA, Chehimi MM. Development of latent fingerprints via aryldiazonium tetrachloroaurate salts on copper surfaces: An XPS study. Langmuir. 2020;36:74-83.

13. Mooste M, Kibena E, Matisen L, Tammeveski K. Blocking properties of nickel electrodes modified with aryldiazonium compounds. Int. J. Electrochem. Sci. 2015;10:3803-3819. 
14. Jacques A, Devillers S, Delhalle J, Mekhalif Z. Electrografting of in situ generated pyrrole derivative diazonium salt for the surface modification of nickel. Electrochim. Acta. 2013;109:781-789.

15. Arrotin B, Jacques A, Devillers S, Delhalle J, Mekhalif Z. Induction heating to trigger the nickel surface modification by in situ generated 4-carboxybenzene diazonium. Appl. Surf. Sci. 2016;370:320-327.

16. Given BW. Latent fingerprints on cartridges and expended cartridge casings. $J$ Forensic Sci. 1976;3:587-594.

17. Aralekallu S, Kuntoji G, Nemakal M, Mohammed I, Sannegowda LK. Self-assembly of reactive difunctional molecules on nickel electrode. Surfaces and Interfaces. 2019;15;1925.

18. Lutterotti L, Vasin R, Wenk HR. Rietveld texture analysis from synchrotron diffraction images. I. Calibration and basic analysis. Powder Diffr. 2014;29:76-84.

19. Gagg CR, Lewis PR, Counterfeit coin of the realm - Review and case study analysis, Eng. Fail. Anal. 2007;14:1144-1152.

20. Lidén C, Skare L, Vahter M. Release of nickel from coins and deposition onto skin from coin handling - comparing euro coins and SEK, Contact Dermatitis. 2008;59:31-37.

21. Hochholdinger S, Marvin L, Arnoux M, Esseiva P, Delémont O. Elemental analysis for profiling counterfeit watches. Forensic Sci. Int. 2019;298;177-185.

22. Hida M, Sato H, Sugawara H, Mitsui T. Classification of counterfeit coins using multivariate analysis with X-ray diffraction and X-ray fluorescence methods. Forensic Sci. Int. 2001;115:129-134.

23. Hida M, Mitsui T, Minami Y. Forensic investigation of counterfeit coins. Forensic Sci. Int. 1997;89:21-26.

24. Nganvongpanit K, Buddhachat K, Piboon P, Euppayo T, Mahakkanukrauh P. Variation in elemental composition of human teeth and its application for feasible species identification. Forensic Sci. Int. 2017;271:33-42.

25. Liu C, Hua Z, Meng X. Profiling of illicit cocaine seized in China by ICP-MS analysis of inorganic elements. Forensic Sci. Int. 2017;276:77-84.

26. Edmiston KE, Johnson J. Determining an optimal sequence for chemical development of latent prints on cartridge casings and shotgun shells. J. Forensic Sci. 2009;54:1327- 1331.

27. Chophi R, Sharma S, Sharma S, Singh R. Trends in the forensic analysis of cosmetic evidence, Forensic Chem. 2019;14:100165. 
28. Bailey MJ, Bright NJ, Croxton RS, Francese S, Ferguson LS, Hinder S, Jickells S, et al. Chemical characterization of latent fingerprints by matrix-assisted laser desorption ionization, time-of-flight secondary ion mass spectrometry, mega electron volt secondary mass spectrometry, gas chromatography/mass spectrometry, X-ray photoelectron spectroscopy, and attenuated total reflection fourier transform infrared spectroscopic imaging: an intercomparison. Anal. Chem. 2012;84:8514-8523.

29. Green FM, Salter TL, Stokes P, Gilmore IS, O'Connor G. Ambient mass spectrometry: advances and applications in forensics. Surf. Interface Anal. 2010;42:347-357.

30. Dafydd H, Williams G, Bleay S. Latent fingerprint visualization using a scanning Kelvin probe in conjunction with vacuum metal deposition. J. Forensic Sci. 2014;59:211-218.

31. Watts JF. The potential for the application of X-ray photoelectron spectroscopy in forensic science. Surf. Interface Anal. 2010;42;358-362.

32. Suzuki EM, McDermot MX. Infrared spectra of U.S. automobile original finishes. VII. Extended range FT-IR and XRF analyses of inorganic pigments in situ-nickel titanate and chrome titanate, J. Forensic Sci. 2006;51:532-547.

33. Roelofse F, Horstmann UE. A case study on the application of isotope ratio mass spectrometry (IRMS) in determining the provenance of a rock used in an alleged nickel switching incident, Forensic Sci. Int. 2008;174:63-67.

34. Cao Y, Nyborg L, Jelvestam U. XPS calibration study of thin-film nickel silicides. Surf. Interface Anal. 2009;41:471-483. 


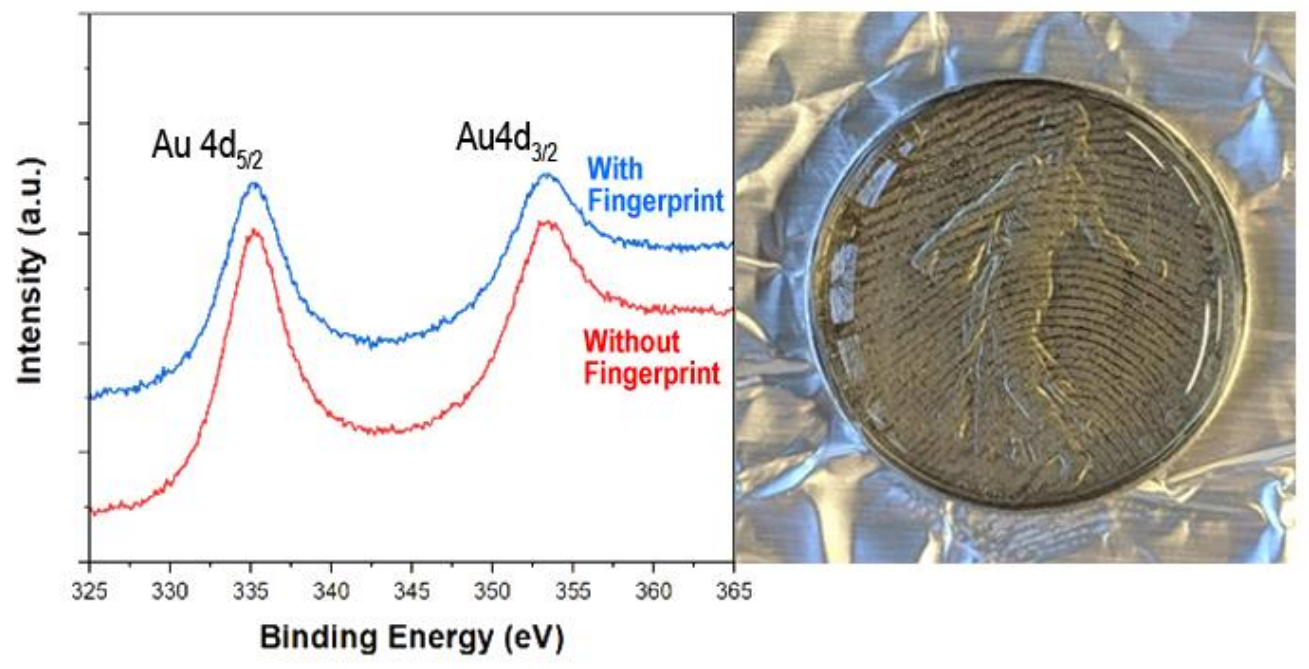




\section{Highlights}

- Reduction of diazonium gold(III) salts occurs spontaneously

- Latent fingerprints can be detected on nickel coins

- XPS proved to be an excellent tool in forensic studies

- Reducible $\mathrm{AuCl}_{4}$ anions are easily reducible on fingerprints

- One step development is presented 